\title{
RADIOMETRIC AND GEOMETRIC CHARACTERISTICS OF PLEIADES IMAGES
}

\author{
K. Jacobsen ${ }^{\mathrm{a}}$, H. Topan ${ }^{\mathrm{b}}$, A.Cam ${ }^{\mathrm{b}}$, M. Özendi ${ }^{\mathrm{b}}$, M. Oruc ${ }^{\mathrm{b}}$ \\ ${ }^{a}$ Leibniz University Hannover, Institute of Photogrammetry and Geoinformation, Germany; \\ ${ }^{\mathrm{b}}$ Bülent Ecevit University, Zonguldak, Turkey \\ jacobsen@ipi.uni-hannover.de; topan@beun.edu.tr, alicam193@gmail.com,mozendi@gmail.com, \\ orucm@hotmail.com
}

\section{ISPRS WG I/4}

KEY WORDS: Space, Adjustment, Restitution, Georeferencing, Radiometric

\begin{abstract}
:
Pleiades images are distributed with $50 \mathrm{~cm}$ ground sampling distance (GSD) even if the physical resolution for nadir images is just $70 \mathrm{~cm}$. By theory this should influence the effective GSD determined by means of point spread function at image edges. Nevertheless by edge enhancement the effective GSD can be improved, but this should cause enlarged image noise. Again image noise can be reduced by image restoration. Finally even optimized image restoration cannot improve the image information from $70 \mathrm{~cm}$ to $50 \mathrm{~cm}$ without loss of details, requiring a comparison of Pleiades image details with other very high resolution space images.

The image noise has been determined by analysis of the whole images for any sub-area with 5 pixels times 5 pixels. Based on the standard deviation of grey values in the small sub-areas the image noise has been determined by frequency analysis. This leads to realistic results, checked by test targets. On the other hand the visual determination of image noise based on apparently homogenous sub-areas results in too high values because the human eye is not able to identify small grey value differences - it is limited to just approximately 40 grey value steps over the available gray value range, so small difference in grey values cannot be seen, enlarging results of a manual noise determination.

A tri-stereo combination of Pleiades 1A in a mountainous, but partially urban, area has been analyzed and compared with images of the same area from WorldView-1, QuickBird and IKONOS. The image restoration of the Pleiades images is very good, so the effective image resolution resulted in a factor 1.0, meaning that the effective resolution corresponds to the nominal resolution of $50 \mathrm{~cm}$. This does not correspond to the physical resolution of $70 \mathrm{~cm}$, but by edge enhancement the steepness of the grey value profile across the edge can be enlarged, reducing the width of the point spread function. Without additional filtering edge enhancement enlarges the image noise, but the average image noise of approximately 1.0 grey values related to 8bit images is very small, not indicating the edge enhancement and the down sampling of the GSD from $70 \mathrm{~cm}$ to $50 \mathrm{~cm}$. So the direct comparison with the other images has to give the answer if the image quality of Pleiades images is on similar level as corresponding to the nominal resolution. As expected with the image geometry there is no problem. This is the case for all used space images in the test area, where the point identification limits the accuracy of the scene orientation.
\end{abstract}

\section{INTRODUCTION}

The main criteria for the comparison of image information content of different sensors is the ground sampling distance (GSD), the distance of neighbored pixel centers projected to object space. The GSD must not correspond to the pixel size in the image multiplied with the scale number because of over- or under-sampling. For oblique space images the geometric pixel size in object space depends upon the incidence angle i (formula 1). The incidence angle is the nadir angle at the ground point to the satellite which is larger as the nadir angle from the satellite to the ground point because of earth curvature.

Ground pixel in view direction: Pview $=$ Pnadir $/\left(\cos ^{2} i\right)$ Ground pixel size across view direction:

$$
\text { Pacross }=\text { Pnadir } / \cos i)
$$

Formula 1: ground pixel size as function of incidence angle $\mathrm{i}$

In the case of a satellite image with $50 \mathrm{~cm}$ ground pixel size in the nadir for $30^{\circ}$ incidence angle, the projected pixel size is $58 \mathrm{~cm} \times 67 \mathrm{~cm}$. For the flying height of Pleiades the incidence angle of $30^{\circ}$ corresponds to a nadir angle at the satellite of $26.8^{\circ}$. The influence to the ground pixel size causes a not negligible loss of ground resolution. In addition for images from a sensor with staggered CCDs, where neighbored pixels in the images are oversampled by $50 \%$, the effective GSD is not the same as the nominal GSD. Nevertheless satellite images often are delivered with the GSD of the nadir even if the effect of nadir angles cannot be neglected. Problems with the image quality may be caused also by the optics, the atmospheric conditions and not satisfying light conditions in case of low sun angle. It can be improved by transfer delay and integration (TDI). The complex situation requires the determination of the effective GSD based on edge analysis (Jacobsen 2008, Jacobsen 2014). In addition the signal to noise relation has to be checked as well as the identification of objects.

\section{ANALYZED DATA}

The comparison of space images is limited by varying imaging conditions. The image quality depends upon available light dominated by the sun elevation. In addition the atmospheric conditions may be different, but it can be improved by TDI. For the test area Zonguldak in Turkey at the Black Sea coast 
changing atmospheric conditions are usually negligible with the exception of clouds and the area close to clouds - such areas have been excluded.

\begin{tabular}{|l|c|c|c|c|}
\hline satellite & imaging & $\begin{array}{c}\text { Sun } \\
\text { elevation }\end{array}$ & $\begin{array}{c}\text { Incidence } \\
\text { angle }\end{array}$ & $\begin{array}{c}\text { Delivered } \\
\text { GSD }\end{array}$ \\
\hline $\begin{array}{l}\text { Pleiades 1A } \\
\text { center image }\end{array}$ & $\begin{array}{c}2013- \\
04-24\end{array}$ & $59.2^{\circ}$ & $5.8^{\circ}$ & $0.5 \mathrm{~m}$ \\
\hline WorldView-1 & $\begin{array}{c}2008- \\
09-16\end{array}$ & $49.7^{\circ}$ & $28.6^{\circ}$ & $0.5 \mathrm{~m}$ \\
\hline QuickBird & $\begin{array}{l}2004- \\
05-23\end{array}$ & $64.7^{\circ}$ & $3.9^{\circ}$ & $0.62 \mathrm{~m}$ \\
\hline IKONOS & $\begin{array}{l}2002- \\
07-02\end{array}$ & $67.2^{\circ}$ & $20.3^{\circ}$ & $1.0 \mathrm{~m}$ \\
\hline
\end{tabular}

Table 1: Analyzed satellite images

The different sun elevations (table 1) are not below a critical limit and are influencing mainly the size of shadow regions in the built up area. The incidence angles are only larger for WorldView-1 and IKONOS. For WorldView-1 the ground pixel size is enlarged by the incidence angle in the average by $21 \%$, but this partially is compensated by the real GSD for nadir images of $0.46 \mathrm{~m}$, corresponding in the average to $0.56 \mathrm{~m}$ physical GSD, up-sampled to $0.50 \mathrm{~m}$. For IKONOS the ground pixel size is enlarged in the average by $10 \%$ which is totally compensated by the real GSD for nadir images of $0.81 \mathrm{~m}$. So the delivered images with $1.0 \mathrm{~m}$ GSD are in fact down-sampled. As mentioned above, Pleiades images are up-sampled from the physical $0.70 \mathrm{~m}$ GSD to the delivered $0.50 \mathrm{~m}$ GSD.

\section{RADIOMETRIC QUALITY}

An object with a sudden change of brightness is imaged with a continuous change of grey values in the image (figure 1, upper part). The grey value profile perpendicular to the edge, averaged over several pixels, shows the continuous grey value change in the image (figure 1, lower left). A differentiation of this profile leads to the point spread function (figure 1, lower right). Half the width of the point spread function corresponds to the factor for the effective resolution; this multiplied with the nominal resolution is identical to the effective resolution, describing the image information content.

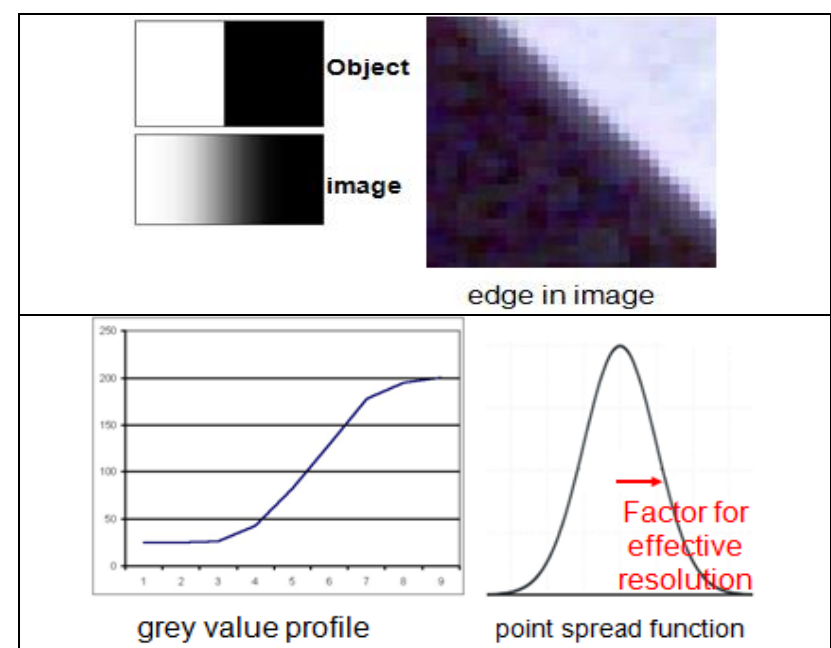

Figure 1: determination of effective image resolution by edge analysis

The determination of the effective resolution by edge analysis can be manipulated by edge enhancement, enlarging the steepness of the edge (figure 3 left $=$ original cross section, figure 3 right after edge enhancement). The edge enhancement improves the contrast (figure 4). This may be an advantage for object identification, but it also increases the image noise. So an edge analysis should be accompanied by noise determination.

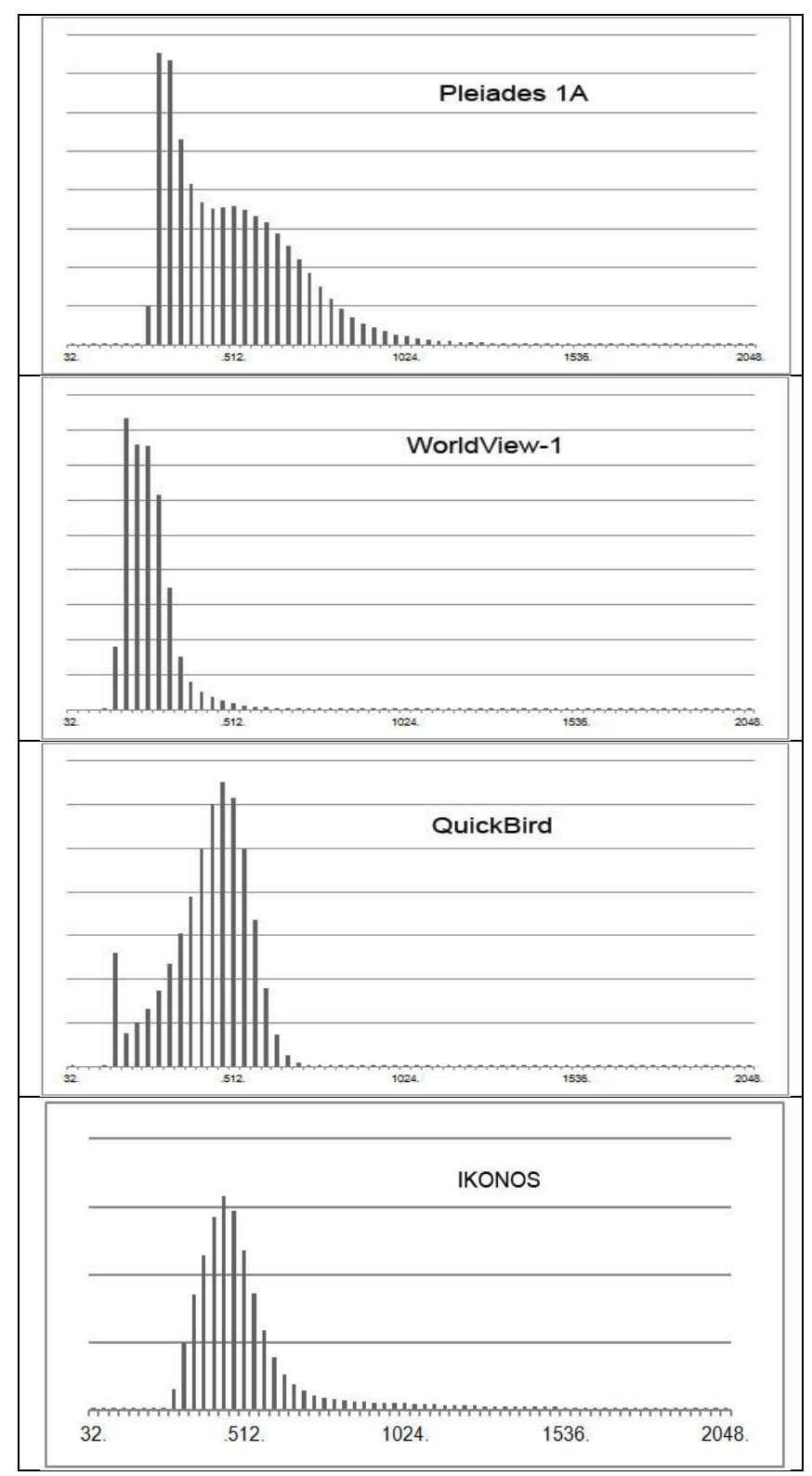

Figure 2: Frequency distribution of grey values in original 16 bit images

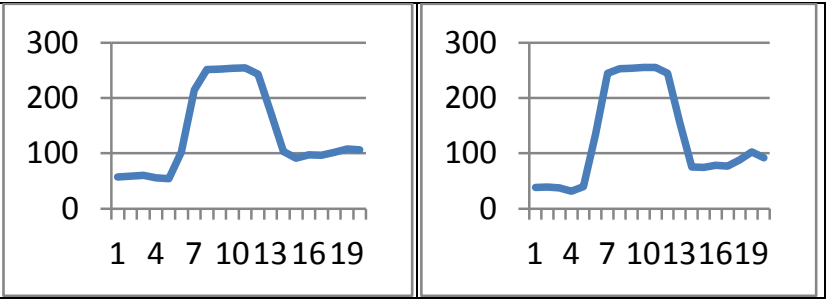

Figure 3: cross section through bright object in Pleiades image vertical $=$ grey value, horizontal $=$ pixel position Left: original image, right: after edge enhancement

A manual noise analysis, for example with Photoshop, leads to too pessimistic results. The human eye is not sensitive enough to identify sub-areas with the same grey value, requiring an 
automation of this analysis. For this reason the standard deviation of the grey values in sub-areas of $5 \times 5$ pixels is computed and by histogram analysis the noise is determined. The noise analysis can be based on the original 16bit images or in the very often used images changed to 8bit resolution.

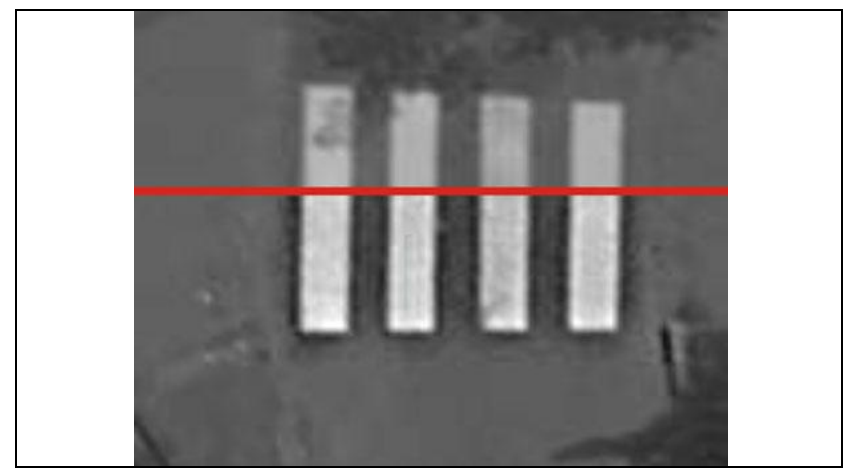

Figure 4: upper part - original aerial image, lower part after strong edge enhancement

Pleiades images as well as the other compared images are defined with 12bit pixel depth, corresponding to 2048 grey values (Pleiades Imagery User Guide) as the other analyzed images. The grey value frequency distribution of the nadir Pleiades image (figure 2 above) shows that not a high number of pixels are in the dark and the bright range. Only $0.04 \%$ of the grey values are in the range up to the grey value 224 and just $0.57 \%$ above 1248 . Even if this may be an advantage for very dark or very bright image parts, the advantage of 12 bit against 11 bit is limited. Also an optimal fit to 8bit grey values ( 256 grey values) did not really reduce the image information. The frequency distribution for IKONOS and QuickBird in the dark and bright parts is similar (figure 2). But in relation to the other images the grey values are better distributed for Pleiades, being an advantage for the object identification (see also figure 5).

\begin{tabular}{|c|c|c|c|}
\hline Pleiades 1A & WorldView-1 & QuickBird & IKONOS \\
\hline 1.00 & 1.02 & 1.01 & 1.02 \\
\hline
\end{tabular}

Table 2: Factor for effective GSD

The factors for the effective GSD, determined by edge analysis, for all four image types is nearly identical to 1.00 . That means the effective ground resolution corresponds to the nominal. For WorldView-1, QuickBird and IKONOS this was expected based on several investigations in other areas (Jacobsen 2011). For Pleiades 1A the up-sampling from $0.7 \mathrm{~m}$ physical GSD to $0.5 \mathrm{~m}$ nominal GSD seems to be justified. That means the radiometric processing of Pleiades is very good. With edge enhanced digital aerial images factors for effective GSD up to 0.76 , but more often 0.85 has been reached (Jacobsen 2014), showing that the factor of the effective resolution can be improved also below the limit of 1.0.

\begin{tabular}{|l|c|c|c|c|}
\hline & \multicolumn{3}{|c|}{8 bit images } & $\begin{array}{c}16 \text { bit } \\
\text { images }\end{array}$ \\
\hline & $\begin{array}{c}\text { Average } \\
\text { noise } \\
\text { [grey } \\
\text { values] }\end{array}$ & $\begin{array}{c}\text { Weighted } \\
\text { noise } \\
\text { [grey } \\
\text { values] }\end{array}$ & SNR & SNR \\
\hline Pleiades 1A & 1.0 & 1.0 & 36.8 & 105.6 \\
\hline WorldView-1 & 2.4 & 1.4 & 26.7 & 68.7 \\
\hline QuickBird & 3.2 & 1.5 & 54.2 & 118.6 \\
\hline IKONOS & 2.7 & 1.0 & 44.1 & 105.5 \\
\hline
\end{tabular}

Table 3: Noise analysis
For satellite images based on staggered CCDs as IRS-1C, SPOT-5 supermode, OrbView-3, Resourcesat and Cartosat-1 the factor for effective GSD is in the range of 1.1 up to 1.2 , clearly indicating that the effective resolution is not as good as the nominal resolution (Jacobsen 2011).

As mentioned above by edge enhancement the image noise usually is enlarged, limiting the strength of edge enhancement. The standard deviation of the grey values (noise) in table 3 is listed for 8 bit images as average for equal distributed grey value groups and as weighted value, corresponding to the frequency. The weighted noise in 8 bit images with 1.0 up to 1.5 grey values is very low and not disturbing the object identification. More informative is the signal to noise relation (SNR), which is satisfying for all four sensor types. The low noise was expected at least for Pleiades because as mentioned in the Pleiades Imagery User Guide, Pleiades images are "deconvoluted, enhancing the image sharpness and de-noised in addition to the zooming" from $0.7 \mathrm{~m}$ GSD to $0.5 \mathrm{~m}$ GSD.

Of course a de-noising may cause an image smoothing so that small details can be lost. That means finally a direct comparison of the images is required. The Federation of American Scientists (FAS) developed the National Image Interpretability Rating Scales (NIIRS) at first for military reconnaissance, but also with the Civil NIIRS Reference Guide (NIIRS 1996) for civil application. The NIIRS tries to quantify images for interpretation into 10 steps from NIIRS 0 (over 9.0m GSD) up to NIIRS 9 (less than $0.10 \mathrm{~m}$ GSD). The level NIIRS 6 corresponds to $0.40 \mathrm{~m}$ up to $0.75 \mathrm{~m}$ GSD. The Civil NIIRS Reference Guide tries to categorize the image quality with agricultural, cultural and natural criteria. The problem and limitation of NIIRS are the included criteria which may fit to the USA, but not for countries as Turkey where most of the criteria are not relevant. In addition the grouping $0.40 \mathrm{~m}$ up to $0.75 \mathrm{~m}$ is too imprecise for above comparison of satellite images, even if the levels are also used with sub-divisions of 0.1 . Finally NIIRS was not helpful for the categorizing of the analyzed images.

Figure 5 shows samples of images taken by Pleiades 1A, WorldView-1, QuickBird and IKONOS. Of course the sun elevation is not exactly the same, but the sun azimuth is close together caused by similar local time of imaging. In addition the atmospheric conditions may be slightly different, limiting the meaning of the comparison. The images have been taken from 2002 up to 2013 (table 1), so some changes in object space exist. Pleiades $1 \mathrm{~A}$ and WorldView-1 are both available with $0.5 \mathrm{~m}$ GSD, but we should remember that the physical GSD of Pleiades is just $0.7 \mathrm{~m}$. Nevertheless the Pleiades image is clearer and allows simpler object identification. Small details as objects on roofs or cars are shown better in the Pleiades as in the WorldView image. Even the QuickBird image with $0.62 \mathrm{~m}$ GSD is more detailed as the WorldView-1 image with 0.5m GSD. In relation to the Pleiades image caused by better contrast the mapping with the QuickBird image is more difficult, but the contents nearly is the same. The available IKONOS image has just $1.0 \mathrm{~m}$ GSD, clearly a lower resolution as for the other images. It belongs to the NIIRS level $5(0.75 \mathrm{~m}-1.2 \mathrm{~m}$ GSD). The IKONOS image is not really worse in relation the other images, but small objects cannot be identified as well. The wider frequency distribution of Pleiades grey values (figure 2) explains the good situation of Pleiades.

As result of the comparison can be stated that the restauration of the Pleiades image is very good, resulting in image information justifying a distribution with $0.5 \mathrm{~m}$ GSD. The signal to noise relation in the original 16 bit images with 105 up to 118 is very similar for all images, only for WorldView-1 with 69 it is not as good. A reason for this may be the sun elevation being for WorldView- $110^{\circ}$ up to $17^{\circ}$ lower as for the other images. 


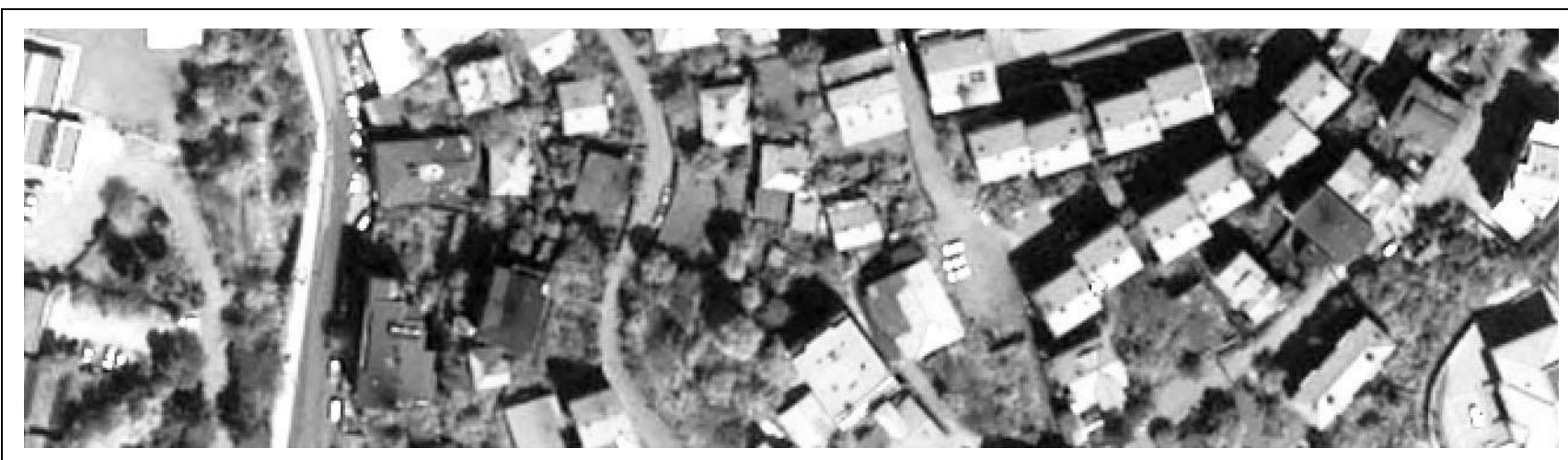

Pleiades 1A, 0.5m GSD, physically 0.7m GSD, imaging at 2013-04-23

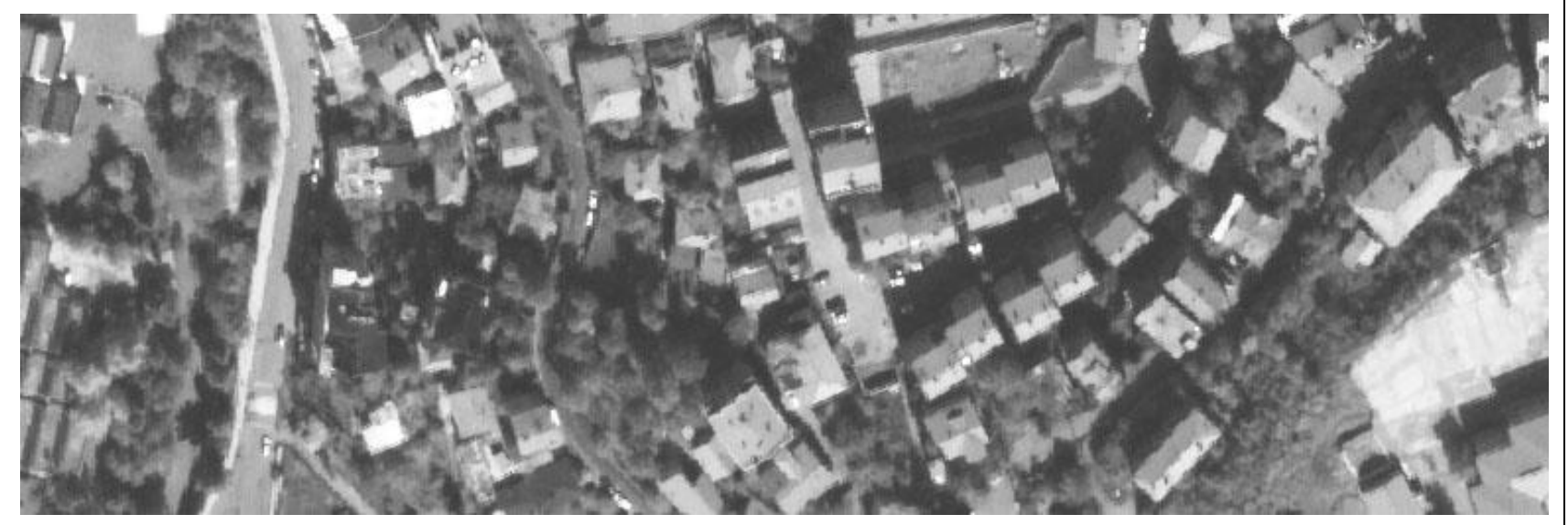

WorldView-1, 0.5m GSD, caused by incidence angle originally $0.56 \mathrm{~m}$ GSD, imaging at 2008-09-16

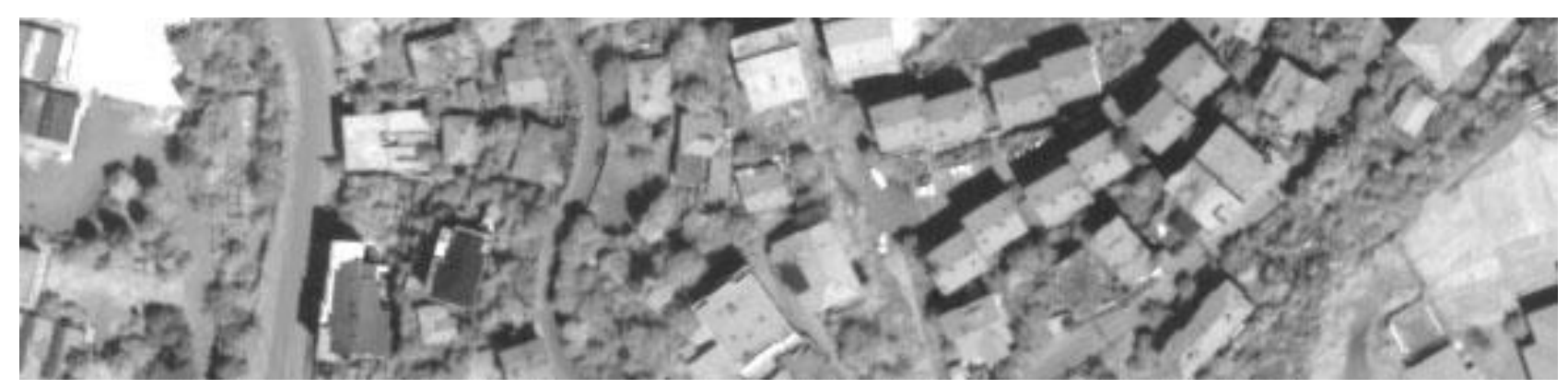

QuickBird, 0.62m GSD, imaging at 2004-05-23

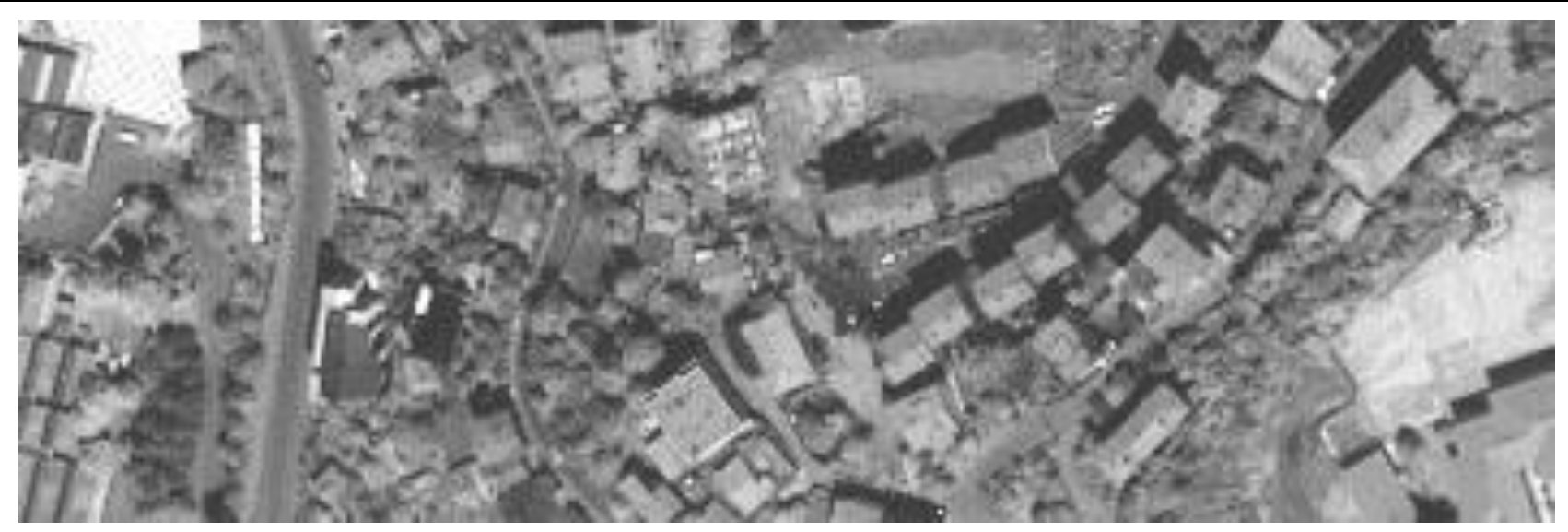

IKONOS, 1m GSD, imaging at 2002-07-02

Figure 5: samples of analyzed images, test area Zonguldak, Turkey, with ground resolution and imaging time 


\section{GEOMETRIC ANALYSIS}

With 163 ground control points (GCP), determined by relative GPS-positioning, root mean square differences as average for $\mathrm{X}$ and $\mathrm{Y}$ of $51 \mathrm{~cm}$ has been reached by scene orientation with Pleiades images in the test area (figure 7). In relation to the physical ground resolution of $70 \mathrm{~cm}$ this corresponds to 0.73 GSD which is a good result for the GCPs mainly defined by edges and not so often symmetric positions. But even in relation to $50 \mathrm{~cm}$ GSD this result is acceptable for the natural ground control points. As shown in figure 6, especially in the rural area the ground control point definition is not optimal, but also in the built up area only a limited number of symmetric points as GSP94 and GCP83 have been used. Several points do not have an optimal location as GCP78 and GCP185. With such points the standard deviation usually cannot be better as 1.0 GSD.

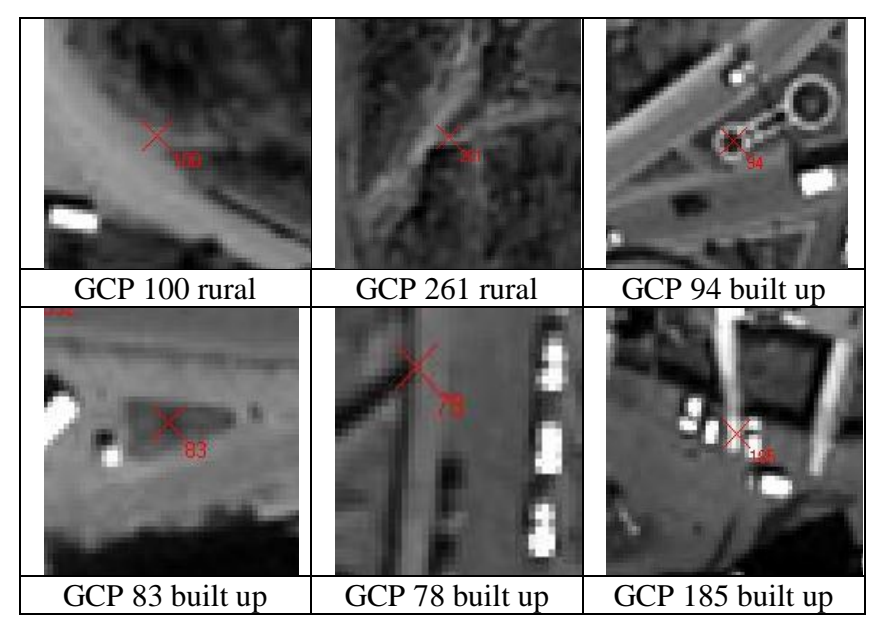

Figure 6: samples of ground control point definitions with information about location in rural or built up area

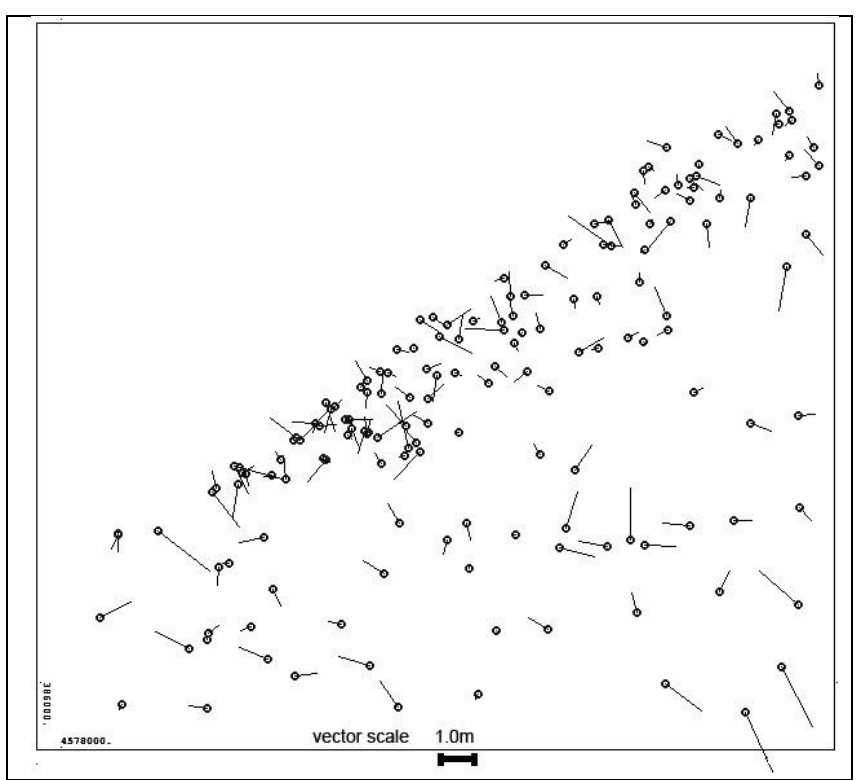

Figure 7: Pleiades 1A image orientation: discrepancies at 163 ground control points, upper left $=$ Black Sea

Sub-pixel accuracy usually only can be reached for symmetric points caused by the not avoidable shift of edges in the image. Discrepancies at neighbored ground control points are correlated below 0.05 meaning that there are no significant systematic errors in the Pleiades scenes as it can be seen in figure 7. 1.0 GSD standard deviation of scene orientation for $\mathrm{X}$ and $\mathrm{Y}$ are realistic under such operational conditions (Jacobsen 2011).

With the other images similar results in relation to the GSD has been reached. The same problems with the ground control point definition exist, but not the same GCP have been used.

\section{CONCLUSION}

In general the results achieved with Pleiades images are acceptable in relation to the distributed image resolution of $0.5 \mathrm{~m}$ GSD even if the physical resolution is just $0.7 \mathrm{~m}$ GSD for nadir images. The edge analysis leads to a factor for the effective resolution of 1.00 in relation to $0.5 \mathrm{~m}$ GSD. The signal to noise relation is on the same level as for QuickBird and IKONOS images and the visual comparison even shows advantages against WorldView-1 image with in the average $0.56 \mathrm{~m}$ physical GSD caused by $28.6^{\circ}$ incidence angle. Also against a QuickBird image with $0.62 \mathrm{~m}$ GSD the Pleiades image has some advantages. Of course IKONOS images with $1.0 \mathrm{~m}$ GSD do not include the same information content. The geometry of Pleiades images is as for most satellite image types without problem, but for operational mapping projects the requirement for image resolution dominates against the geometric request.

Concluding can be stated that it is justified to distribute Pleiades images with $0.5 \mathrm{~m}$ GSD. The radiometric quality is good and the frequency distribution of the grey values has advantages against the compared other space images.

\section{REFERENCES}

Jacobsen, K., 2008: Tells the number of pixels the truth? Effective Resolution of Large Size Digital Frame Cameras, ASPRS 2008 Annual Convention, Portland

Jacobsen, K., 2011: Characteristics of very High Resolution Optical Satellites for Topographic Mapping, ISPRS Hannover Workshop 2011, 6pages, IntArchPhRS Vol XXXVIII-4/W19

Jacobsen, K., 2014: Bildqualität, 34. WissenschaftlichTechnische Jahrestagung der DGPF, DGPF Tagungsband 23 / 2014 Beitrag http://www.dgpf.de/neu/Proc2014/index.html

NIIRS 1996: Federation of American Scientists (FAS), National Image Interpretability Rating Scales, (March 1996), http://fas.org/irp/imint/niirs.htm (October 2014)

Pleiades Imagery User Guide, http://www.astriumgeo.com/en/4572-pleiades-technical-documents (October 2014) 